\title{
A Real-Time Timetable Rescheduling Method for Metro System Energy Optimization under Dwell-Time Disturbances
}

\author{
Guang Yang $\mathbb{D}^{1},{ }^{1}$ Junjie Wang $\mathbb{D}^{1},{ }^{1}$ Feng Zhang $\mathbb{D}^{1},{ }^{1}$ Shiwen Zhang $\mathbb{D}^{1},{ }^{1}$ and Cheng Gong $\mathbb{D}^{2}$ \\ ${ }^{1}$ School of Electronic Information and Electrical Engineering, Shanghai Jiao Tong University, Shanghai 200240, China \\ ${ }^{2}$ School of Electrical and Computer Engineering, Georgia Institute of Technology, Atlanta, Georgia 30332, USA \\ Correspondence should be addressed to Feng Zhang; fzhang@sjtu.edu.cn
}

Received 15 April 2019; Accepted 17 August 2019; Published 2 December 2019

Academic Editor: Rakesh Mishra

Copyright (C) 2019 Guang Yang et al. This is an open access article distributed under the Creative Commons Attribution License, which permits unrestricted use, distribution, and reproduction in any medium, provided the original work is properly cited.

\begin{abstract}
Automatic Train Systems (ATSs) have attracted much attention in recent years. A reliable ATS can reschedule timetables adaptively and rapidly whenever a possible disturbance breaks the original timetable. Most research focuses the timetable rescheduling problem on minimizing the overall delay for trains or passengers. Few have been focusing on how to minimize the energy consumption when disturbances happen. In this paper, a real-time timetable rescheduling method (RTTRM) for energy optimization of metro systems has been proposed. The proposed method takes little time to recalculate a new schedule and gives proper solutions for all trains in the network immediately after a random disturbance happens, which avoids possible chain reactions that would attenuate the reuse of regenerative energy. The real-time feature and self-adaptability of the method are attributed to the combinational use of Genetic Algorithm (GA) and Deep Neural Network (DNN). The decision system for proposing solutions, which contains multiple DNN cells with same structures, is trained by GA results. RTTRM is upon the foundation of three models for metro networks: a control model, a timetable model and an energy model. Several numerical examples tested on Shanghai Metro Line 1 (SML1) validate the energy saving effects and real-time features of the proposed method.
\end{abstract}

\section{Introduction}

With rapid development of intelligent transportation, Automatic Train Systems (ATSs) have attracted much attention in recent years. A reliable high-level ATS ensures the entire railway network to function safely, cost-effectively and efficiently under sudden disturbances [1]. Disturbances in metro networks such as temporary platform blockages make offline schedules suboptimal for use. Hence, various methods of Train Timetable Rescheduling (TTR) [2-6] have been proposed to handle unforeseen events which may disturb timetable. Prevailing methods for timetable rescheduling can be classified into two categories: passenger-oriented and train-oriented. The passenger-oriented research focuses on minimizing the total delay time of passengers [4], while the train-oriented research focuses on minimizing the overall delays of all trains $[7,8]$. However, short delays in one train may not necessarily arouse block conflicts of the route ahead, still, it will inevitably affect the energy consumption of the overall metro network. Since a metro network consumes a large amount of electric energy every day, there is tremendous potential of energy conservation in metro transportations. So far, there is little research that mentions the topic on how to save energy when a metro network encounters disturbances.

Different from the traditional TTR problem, the energy-efficient TTR problem consists of two steps. The first step is to build an offline timetable for optimal use of the total energy of the metro network, which is called Energy-Efficient Train Timetabling (EETT) [9]. In this step, all trains in the network run with confirmed dwell time and no emergencies happen [8]. The second step is to build a real-time timetable to cope with sudden disturbances. In this step, proper travel time in different sections and different trains will be determined and modified in real time to minimize the system energy consumption after disturbances happen.

Energy-efficient TTR problem is a challenging task because of its real-time demand and randomness of disturbances. Any disturbance in a metro network can bring a series of chain reactions that make the offline schedule not optimal any more. Rescheduling timetable is a good solution to avoid 
the chain reactions and save more energy. EETT methods that are nonreal-time methods cannot be used in the energy-efficient TTR problem. Moreover, disturbances occur randomly at different stations on different trains, and the length of delay is also stochastic. It requires the method having a self-adaptability that makes the appropriate choice presciently according to the disturbances.

Energy-efficient TTR problem has been rarely mentioned in recent years. Gong et al. [10] proposed an integrated Energy-efficient Operation Methodology (EOM) to compensate dwell time disturbances in real-time. By reducing the travel time of the delaying train in the section following a disturbance, the schedule can be recovered to the original one which is optimized by GA. The cost of EOM is that more energy will be consumed before the recovery of the schedule.

In this paper, a real-time timetable rescheduling method (RTTRM) for energy optimization is proposed. Different from EOM, this method reschedules all trains in the network after a disturbance happens, rather than reschedules the delaying train only. The proposed method can finish making decisions immediately after a disturbance happens, attributed to the combinational usage of GA and DNN. Firstly, GA provides a series of corresponding energy-optimal timetables for random disturbances. Then, a decision system based on DNN learns from the connection between the random disturbances and the optimal timetable which are deduced by GA. Finally, the well trained decision system provides proper solutions in different cases of disturbances in real-time.

The remainder of the paper is organized as follows. Section 2 reviews the previous contributions related to TTR and EETT problem. Section 3 presents the description of a control model, a timetable model and an energy model in metro system. Section 4 introduces GA and a decision system to solve the energy-efficient TTR with dwell-time disturbances. In section 5 , several experiments based on a real-world network of Shanghai Metro Line 1 (SML1) is presented to validate the proposed method. The final section concludes the paper.

\section{Literature Review}

Pioneering theoretical works related to TTR problem were first carried out by Carey's group [11-13]. They eliminated the distribution and propagation of the train delay by using the sequential solution procedures. Törnquist and Persson [14] presented a Mixed Integer Programming (MIP) model that took into account the reordering and rerouting of trains. D'Ariano et al. [15] computed a conflict-free train timetable compatible with the actual status of the railway network and proposed a branchand-bound algorithm for minimizing the global secondary delay. Khan and Zhou [16] developed a stochastic optimization formulation with the purpose of minimizing the total trip time in a published timetable and reducing the expected schedule delay. Cacchiani and Toth [17] classified approaches of timetable rescheduling into six categories: stochastic optimization [18], light robustness [19], recovery robustness [20], delay management [21], bicriteria and Lagrangian-based approaches [22] and meta-heuristics [23]. Dündar and Sahin [24] developed a
Genetic Algorithm (GA) to reschedule the trains on a single track railway line in Turkey and determined the meets and passes of the trains in the opposite direction. They designed benchmarking experiments among an Artificial Neural Network (ANN), a MIP model and two kinds of GA. They also validated that GA outperforms the other methods. Šemrov et al. [25] used a rescheduling method based on reinforcement learning, more specifically Q-learning, on a real-world railway network in Slovenia. They illustrated that Q-learning leaded to rescheduling solutions that were at least equivalent and often superior to the simple First-In-First-Out (FIFO) method and the random walk method. Xu et al. [7] proposed a Mixed-Integer Linear Program (MILP) model for the quasi-moving block signaling system to reduce the final delay and to solve a real-world instance in China. They optimized traffic in transition from a disordered condition to a normal condition and analyzed delays in different transition phases. Ortega et al. [4] proposed a biobjective optimization method for timetable rescheduling during the end-ofservice period of a subway, in order to minimize the total transfer waiting time for all transfer passengers and the deviation from the scheduled timetable.

Albrecht and Oettich [26] first discussed EETT problem. They used a Dynamic Programming (DP) to calculate the optimal timetable. The quality criteria of the multiobjective optimization function were the overall waiting time and the energy consumption. Albrecht [27] considered to change the additional running time to the synchronize acceleration and the regenerative braking in order to minimize total energy consumption and power peaks. It was the first to study EETT with the regenerative braking. Yang et al. [28] first described EETT problem in a mathematical programming mode and solved this problem by maximizing time overlaps of nearby accelerating and braking trains. Sun et al. [29] developed a bi-objective timetable optimization model to minimize the total passenger waiting time and energy consumption.

\section{Formulation of Model for Metro Network}

In this section, three models are formulated: a control model, a timetable model, and an energy model, all of which are used to describe the operation process for a metro network. For the single-train control model, state variables of trains, such as the position and the speed, are formulated. For the timetable model, the departure time and the arrival time for each single train are calculated. For the energy model, energy exchange among trains running in the same network is formulated.

3.1. Parameter and Variable. The notation system which includes parameters and variables, is presented as follows:

$\beta_{1}$ : conversion efficiency of the train traction system (from electricity to mechanical energy).

$\beta_{2}$ : conversion efficiency of the train braking system (from mechanical energy to electrical energy).

$\beta_{3}$ : feedback coefficient on braking energy.

$N$ : total number of trains in the metro network.

$M$ : total number of stations.

$\gamma: \quad$ index for train, $\gamma \in\{1,2, \ldots, N\}$. 
$\eta: \quad$ index for station, $\eta \in\{1,2, \ldots, M\}$.

$t_{d}^{\eta+1, \gamma}:$ dwell time of train $\gamma$ at station $\eta+1$.

$t_{p}^{\eta, \gamma}:$ departure instants of train $\gamma$ at station $\eta$.

$t_{h}^{\gamma}$ : headway of train $\gamma$.

$t_{s}^{\eta, \gamma}:$ interstation running time of train $\gamma$ running from station $\eta$ to station $\eta+1$.

$T_{s}$ total travel time.

$u_{a}$ : constant traction force in the constant force accelerating phase.

$u_{b}$ : constant braking force in the constant force braking phase.

$v_{a}$ : speed in switching point (from the constant force accelerating phase to the constant power accelerating phase).

$v_{b}$ : speed in switching point (from the constant power braking phase to the constant force braking phase).

$v_{c}^{\eta, \gamma}:$ cruising speed of train $\gamma$ running from station $\eta$ to station $\eta+1$.

$v_{\lim 1}:$ the maximum speed limit between stations.

$v_{\lim 2}:$ the pull-in speed limit and the pull-out speed limit.

$x_{s}^{\eta}: \quad$ spacing of the section $(\eta, \eta+1)$.

$x^{\gamma}: \quad$ current position of train $\gamma$.

$t^{\gamma}: \quad$ current time of train $\gamma$.

$v^{\gamma}: \quad$ current driving speed of train $\gamma$.

$u^{\gamma}$ : traction or braking force per unit mass of train $\gamma$.

$r$ : resistance per unit mass.

$g$ : gravity per unit mass.

\subsection{Assumptions}

(i) The network in the model has narrow spacing (for example Shanghai Metro Line 1). Trains drive in each section in a sequence of accelerating-cruising-braking, without the repeated accelerating and braking.

(ii) The adjacent trains run with enough spacing, so we do not consider the blocking conflict during operation.

(iii) The disturbances are small enough which will not lead to network disruption.

(iv) Only one disturbance happens during a complete test procedure from the first train's departure to the last train's arrival.

(v) The disturbances will not happen at the starting station.

3.3. Control Model for a Single Train. When driving a train from $t=0$ to $t=T_{s}$, according to Newton's Second Law, the motion equations are

$$
\begin{gathered}
\frac{d x}{d t}=v, \\
\frac{d v}{d t}=u-r+g,
\end{gathered}
$$

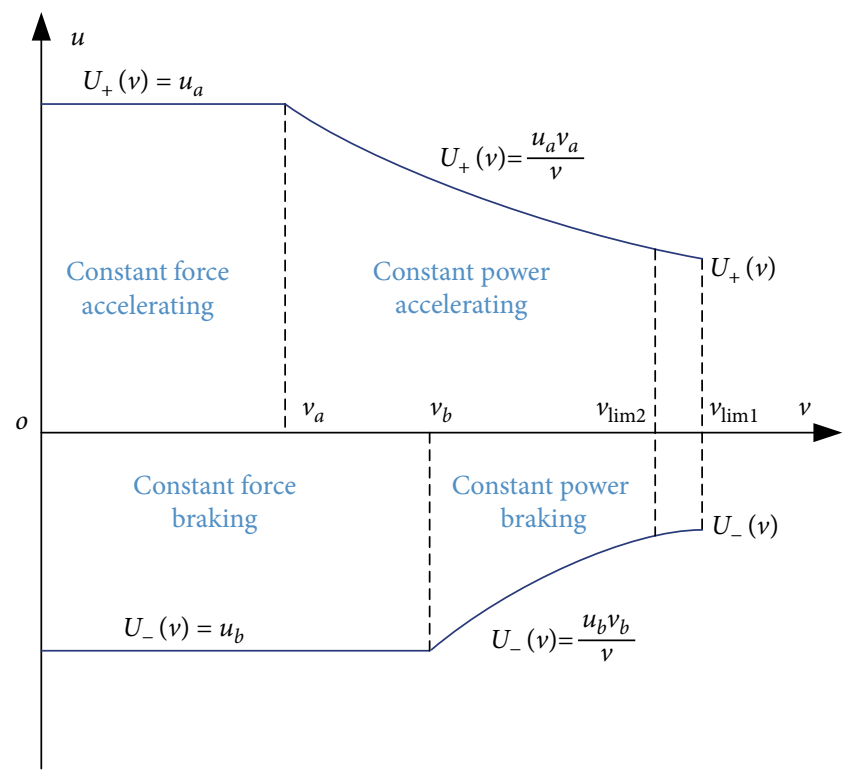

FIGURE 1: The bound curves of accelerating phase and braking phase.

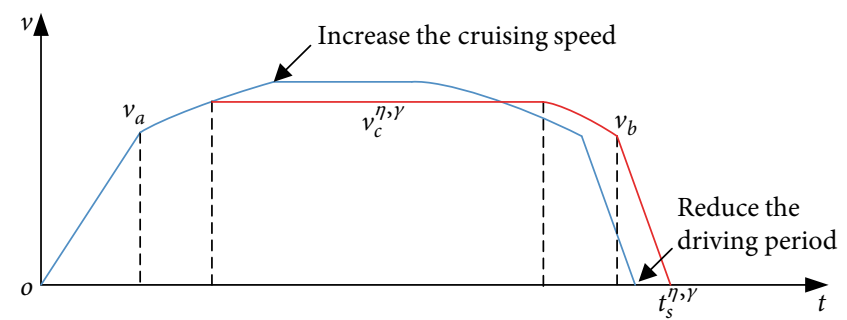

FIGURE 2: The relation between the cruising speed and the periodic time.

where $(x, v)=\left(x^{\gamma}(t), v^{\gamma}(t)\right)$ for $\gamma \in\{1,2, \ldots, N\}$ and $t \in\left[0, T_{s}\right]$. In Equation (2), $r=r(v)$ meets Davis's Equation [30]

$$
r(v)=\lambda_{1} v^{2}+\lambda_{2} v+\lambda_{3},
$$

where $\lambda_{1}, \lambda_{2}$ and $\lambda_{3}$ are constant parameters. $g=g(x)$ represents the gravity per unit mass, which is a piecewise linear function. $u=u^{\gamma}(v, t) \in\left[U_{-}(v), U_{+}(v)\right]$ represents the force per unit mass or acceleration. We assume that $u$ is positive in the acceleration phase and negative in the braking phase. Figure 1 shows the bound curves of $u$, where $U_{-}(v)$ is the lower bound and $U_{+}(v)$ is the upper bound.

In Figure $1,\left(u_{a}, u_{b}, v_{a}, v_{b}, v_{\lim 1}, v_{\lim 2}\right)$ are constants formulated in advance which obey the motor characteristic of a train. According to the theories proposed by the SCG group [31], the maximum acceleration-speedholdmaximum braking strategy is chosen as the optimal driving strategy in a period. The traction force meets $u=U_{+}(v)$ in the accelerating phase, and the braking force meets $u=U_{-}(v)$ in the braking phase. In the speedhold phase, the traction force changes with gradient to keep the driving speed constant, which meets the constraint $U_{-}(v)<u<U_{+}(v)$. According to the functional curves in Figure 1, the expression of variable $u$ in six different phases is concluded as follows. 


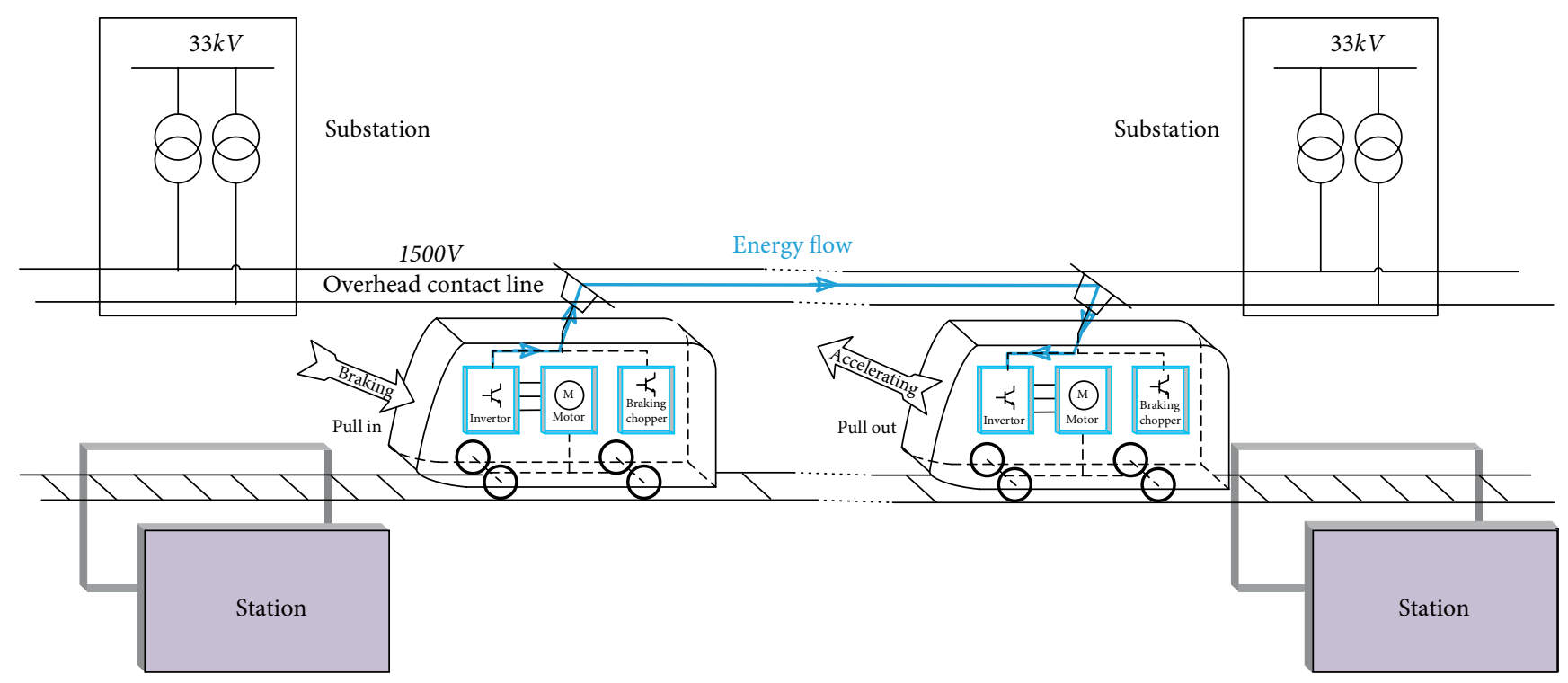

FIGURE 3: Energy exchange between two trains.

(a) Constant force accelerating phase: $u=u_{a}, u_{a}>0$

(b) Constant power accelerating phase: $u=\left(u_{a} v_{a}\right) / v$

(c) Speedhold phase: $u=r(v)-g(x)$

(d) Constant power braking phase: $u=\left(u_{b} v_{b}\right) / v, u_{b}<0$

(e) Constant force braking phase: $u=u_{b}$

(f) Dwelling phase: $u=0$

The numerical examples based on the data of Shanghai Metro Line 1, a network with narrow spacing [10]. Trains drive in each section in a sequence of accelerating-cruising-braking, without the repeated accelerating and braking. The cruising speed $v_{c}^{\eta, \gamma}$ has a one-to-one mapping from the periodic time $t_{s}^{\eta, \gamma}$. The relation is defined as $t_{s}^{\eta, \gamma}=f\left(v_{c}^{\eta, \gamma}\right)$. As shown in Figure 2, the area enclosed by the red curve and the axis $t$ represents the section spacing. If the cruising speed increases, the periodic time will decrease to keep the section spacing constant. Hence, the arrival time can be deduced from the cruising speed.

The derivation process of $t_{s}^{\eta, \gamma}=f\left(v_{c}^{\eta, \gamma}\right)$ is shown in Appendix A.

3.4. Timetable Model for a Single Train. The timetable of a metro-line operation can be modeled as [32]

$$
t_{p}^{\eta+1, \gamma}=t_{p}^{\eta, \gamma}+t_{s}^{\eta, \gamma}+t_{d}^{\eta+1, \gamma},
$$

where $t_{p}^{\eta, \gamma}=t_{h}^{\gamma}$ at $\eta=1$.

If a disturbance occurs in a dwell time $t_{d}^{\eta+1, \gamma}$, then the departure instants will be

$$
t_{p}^{\eta+1, \gamma}=t_{p}^{\eta, \gamma}+t_{s}^{\eta, \gamma}+t_{d}^{\eta+1, \gamma}+\varepsilon .
$$

The maximum travel time for whole trains in the metro-line is

$$
T_{s}=t_{h}^{N}+\sum_{\eta=1}^{M-1}\left(t_{s}^{\eta, N}+t_{d}^{\eta+1, N}\right)+\varepsilon
$$

where $T_{s}$ also represents the total simulation period.

3.5. Energy Model for Multiple Trains. Before considering a case of multitrain driving in one network, one should first consider energy regeneration from braking trains. The braking process converts mechanical energy into electric energy through the overhead contact line. Taking full advantage of this feedback energy can save a large amount of energy. In most common cases, the feedback energy from braking trains is fully utilized by accelerating trains at the same time period. There is another case that energy supply exceeds the demand, and extra energy is consumed by the heating resistors to avoid the voltage increase on the DC side. With the theory shown in Figure 3, it is possible to build an optimal timetable to maximize the utilization of the feedback energy.

Overall energy consumption of a network is derived as follows. Calculate the required traction energy and the regenerated braking energy for train $\gamma$. The traction power at the time can be calculated by

$$
P_{T}^{\gamma}(t)= \begin{cases}\frac{u v}{\beta_{1}} & u<0 \\ 0 & u \leq 0,\end{cases}
$$

where the first condition denotes train $\gamma$ driving in the accelerating phase or in the speedhold phase, and the second condition denotes train $\gamma$ driving in the braking phase or in the dwelling phase. Hence, by summing over the traction power for train and integrating it for time, total required traction energy for all the trains in the network is acquired

$$
E_{T}=\int_{0}^{T_{s}} \sum_{\gamma=1}^{N} P_{T}^{\gamma}(t) d t
$$

The braking energy from train $\gamma$ at time $t$ is

$$
P_{B}^{\gamma}(t)=\left\{\begin{array}{cc}
0 & u>0 \\
-u v \beta_{2} & u \leq 0 .
\end{array}\right.
$$


The total regenerated energy utilized at time $t$ is the minimum between the braking energy feedback and the traction energy, which is

$$
P_{R}(t, \varepsilon)=\min \left(\sum_{\gamma=1}^{N} P_{T}^{\gamma}(t), \beta_{3} \sum_{\gamma=1}^{N} P_{B}^{\gamma}(t)\right) .
$$

The minimum operator is used to distinguish two different conditions. If the traction energy is larger than the regenerative braking energy, the regenerative braking energy will be fully utilized; otherwise if the traction energy is smaller than the regenerative energy, the extra regenerative braking energy will be consumed by the heating resistors. The total regenerative energy for all the trains in the network is

$$
E_{R}(\varepsilon)=\int_{0}^{T_{s}} P_{R}(t, \varepsilon) d t .
$$

The net energy consumption for all the trains in the network is

$$
E(\varepsilon)=E_{T}-E_{R}(\varepsilon)
$$

The objective of energy optimization is to minimize $E(\varepsilon)$.

\section{Energy Optimization with Dwell Time Disturbance}

The objective of the energy-efficient TTR problem is to minimize energy consumption by taking full advantage of the regenerative braking energy when random disturbances happen. In this paper, a real-time timetable rescheduling method (RTTRM) is proposed to solve the energy-efficient TTR problem, which combines GA with a decision system, and gives a proper decision immediately after a random disturbance happens. RTTRM solves the energy-efficient TTR problem in three steps. Firstly, it solves the energy-optimal timetable under a no-disturbance situation. Secondly, by introducing a stochastic variable $\varepsilon$ into the dwell time, ensembles of energy-optimal timetable under different disturbances are traversed. A decision system based on DNN is trained by the state variables which are sampled under different disturbances. In this step, the schedule before the disturbance instant should not be modified. Lastly, the trained decision system is used to judge which case the disturbance belong to and give an energy-optimal solution accordingly.

4.1. Energy Optimization under No-Disturbance Situation. The energy optimization model mentioned in the first step can be formulated as follows:

$$
\left\{\begin{array}{ll}
\min & E(\varepsilon=0) \\
\text { s.t. } & \eta \in(1,2, \ldots, M-1), \gamma \in(1,2, \ldots, N) \\
& t_{p}^{\eta+1, \gamma}=t_{p}^{\eta, \gamma}+t_{s}^{\eta, \gamma}+t_{d}^{\eta+1, \gamma} \\
& t_{s}^{\eta, \gamma}=f\left(v_{c}^{\eta, \gamma}\right) \\
& v_{c \min } \leq v_{c}^{\eta, \gamma} \leq v_{c \max }
\end{array} .\right.
$$

This is a one-objective optimization problem with complex constraints that can be appropriately solved by GA. GA is a stochastic search method for optimization problems inspired by the process of natural selection. Holland [33] first used it to generate high-quality solutions to optimization problems. With extensive generality and practical applicability, it has obtained considerable success in providing satisfactory solutions to many management problems for railway traffic. In this paper, GA is used to solve the energy optimization model.

4.1.1. Genotype. The decision variable in this energy optimization problem is $v_{c}^{\eta, \gamma}$, a continuous variable changing from $v_{c \min }$ to $v_{c \max }$. It is difficult for a genotype covering the range of the continuous decision variable. Hence, we discrete the $\left[v_{c \min }, v_{c \max }\right]$ section and define a genes set $g=\left\{g_{i} \mid g_{i}=0,1,2, \ldots, \theta\right\}$ to correspond the possible values of the decision variable, where $\theta$ is a positive integer. The possible values of the decision variable is

$$
v_{c i}=v_{c \min }+\frac{v_{c \max }-v_{c \min }}{\theta} \cdot g_{i}
$$

4.1.2. Chromosomes. A $(M-1) \times N$-dimensional matrix is used to define a chromosome, in which $c_{(\eta, \gamma)}$ is selected from the genes set $g$.

$$
c=\left\{c_{(\eta, \gamma)} \mid \eta \in[1, M-1], \quad \gamma \in[1, N], \quad c_{(\eta, \gamma)} \in g\right\} .
$$

4.1.3. Initialization. An integer number pop_size is defined as the population size of the initial population. pop_size decreases during each successive generation with genetic operators like crossover and mutation. $E(\varepsilon=0)$ is defined as the fitness function of every individual. An evolutionary computation framework based on python named DEAP is used, which contains GA and other advanced evolutionary strategies. The process of crossover and mutation in GA can be referred to the official documents on Github [34].

4.2. Energy Optimization under Disturbance Situation. The optimization model mentioned in the second step is formulated as follows:

$$
\left\{\begin{array}{lll}
\min & E(\varepsilon) \\
\text { s.t. } & \varepsilon_{\min } \leq \varepsilon \leq \varepsilon_{\max } & \\
& \forall\left(\eta_{0}, \gamma_{0}\right), \eta_{0} \in(1,2, \ldots, M-2), & \gamma_{0} \in(1,2, \ldots, N) \\
& t_{p}^{\eta+1, \gamma}=t_{p}^{\eta, \gamma}+t_{s}^{\eta, \gamma}+t_{d}^{\eta+1, \gamma}+\varepsilon & \text { if } \eta=\eta_{0}, \gamma=\gamma_{0} \\
& t_{p}^{\eta+1, \gamma}=t_{p}^{\eta, \gamma}+t_{s}^{\eta, \gamma}+t_{d}^{\eta+1, \gamma} & \text { others } \\
& t_{s}^{\eta, \gamma}=f\left(v_{c}^{\eta, \gamma}\right) & \\
& v_{c \min } \leq v_{c}^{\eta, \gamma} \leq v_{c \max }
\end{array}\right.
$$

where $\varepsilon$ is not zero but a stochastic variable. GA is also used to solve the energy optimization problem based on (16). In this situation, the chromosome is not a $(M-1) \times N$ dimensional matrix. The dimension of the chromosome is determined by the value of $\left(\eta_{0}, \gamma_{0}\right)$. A delay occurs during the time interval $\left[t_{p}^{\eta, \gamma}, t_{p}^{\eta, \gamma}+t_{d}^{\eta+1, \gamma}+\varepsilon\right]$, so the timetable rescheduling task begins after the instant $t_{p}^{\eta, \gamma}+t_{d}^{\eta+1, \gamma}+\varepsilon$. The optimal independent variables are taken as the reference. Before the delay happens, the original $v_{c}^{\eta, \gamma}$ deduced from (13) are retained. $v_{c}^{\eta, \gamma}$ after the delay are regarded as the new independent variables for (16). 


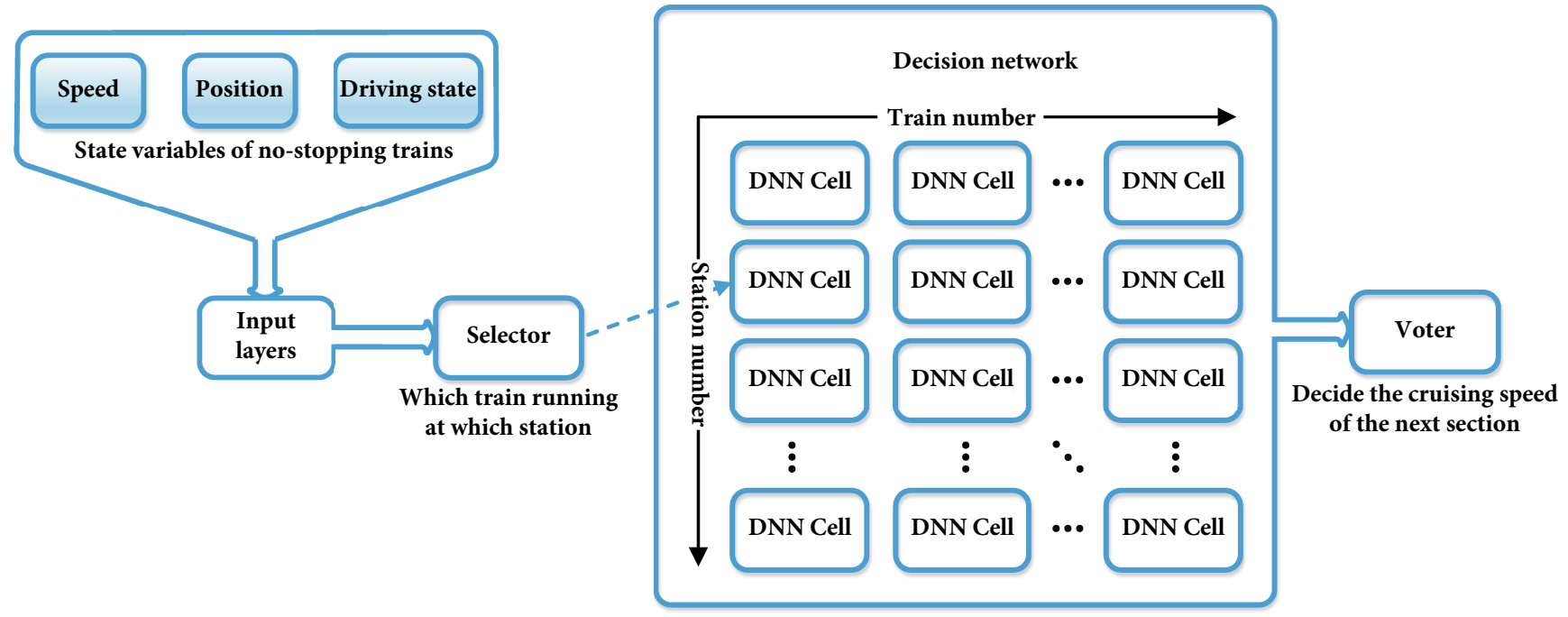

(a)

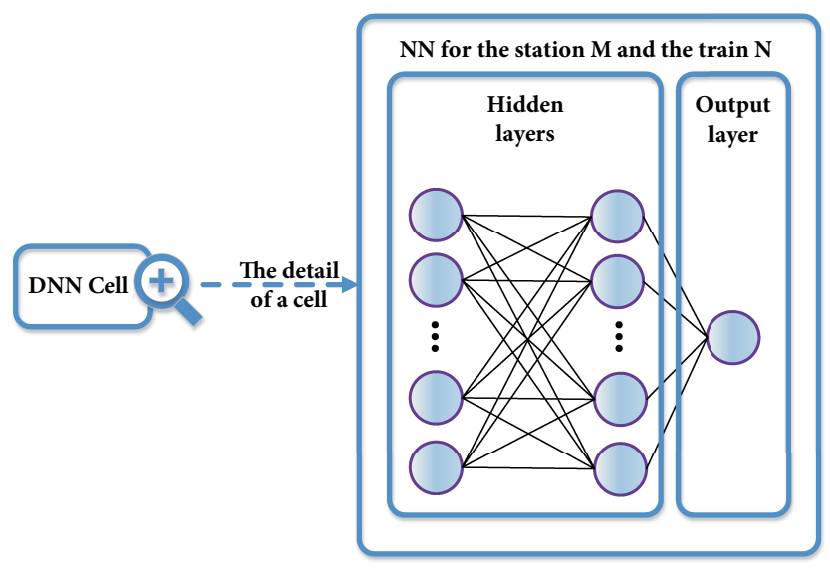

(b)

FIgUre 4: Cruising speed decision system. (a) Structure of decision system and (b) Structure of the DNN cell.

4.3. Deep Neural Network. Deep Neural Network (DNN) [35] has strong generalization ability, and has been widely applied to fields including computer vision, speech recognition, natural language processing, audio recognition, and board game programs, where they have produced results comparable to and in some cases superior to human experts. Compared with Shallow Neural Network (SNN) [36], DNN has an excellent ability of feature learning, and the acquired characteristics can describe the nature of the samples, which is beneficial to visualization or classification. The sample capacity and generalization ability of DNN have been greatly improved than SNN.

In the previous section, a series of sampling data by GA are obtained, which contains the state variables of all trains in the network, sampling at each departure instant in different disturbances, and also contains a series of cruising speed as the target of decisions. These data can be used to train a decision system based on DNN which gives wise choice of cruising speed when disturbances happen. Figure 4 displays a structure of the decision system, which decides cruising speed at each departure instant.

Figure 4(a) shows the structure of the cruising speed decision system, which contains four parts: an input layers, a selector, a decision network and a voter. Cruising speed decision system comes into effect after a disturbance occurs. At the departure instant of each train, the decision system decides the cruising speed of that train in the next section. The state variables of other running trains are put into the input layers. Selector is used to select corresponding DNN cell according to the train number and the station number at present of the departing train, which effectively simplifies the structure of DNN cell. Finally, voter decides the cruising speed of the departing train. Figure 4(b) shows the detailed structure for one of the DNN cell, which is trained by GA results at each disturbance case. DNN cell is structured according to the existing sample volume. Theoretically, the disturbance cases are infinite, so the samples obtained by GA are infinite. Hence, DNN but not SNN is needed to ensure the sample capacity of the decision system.

\section{Experimental Validation}

In order to validate the real-time timetable rescheduling method, a series of numerical experiments are set. The environment for experiments is set in Shanghai Metro Line 1 
TABLE 1: Optimal timetable of the test segment without disturbance.

\begin{tabular}{|c|c|c|c|c|c|c|c|}
\hline Section & Spacing $[\mathrm{m}]$ & Train No. & $\begin{array}{l}\text { Dwell time/ } \\
\text { headway [s] }\end{array}$ & $\begin{array}{c}\text { Accelerating } \\
\text { time }[\mathrm{s}]\end{array}$ & $\begin{array}{c}\text { Cruising time } \\
{[\mathrm{s}]}\end{array}$ & $\begin{array}{c}\text { Braking time } \\
{[\mathrm{s}]}\end{array}$ & $\begin{array}{c}\text { Cruising speed } \\
{[\mathrm{m} / \mathrm{s}]}\end{array}$ \\
\hline \multirow{2}{*}{$\begin{array}{l}\text { Xinzhuang to } \\
\text { waihuan road }\end{array}$} & \multirow{2}{*}{1458.5} & 1 & 0 & 26.5 & 43.0 & 19.5 & 21.36 \\
\hline & & 2 & 120 & 20.1 & 61.4 & 16.3 & 18 \\
\hline \multirow{2}{*}{$\begin{array}{l}\text { Waihuan road to } \\
\text { lianhua road }\end{array}$} & \multirow{2}{*}{1125.7} & 1 & 20 & 27.3 & 25.8 & 19.9 & 21.72 \\
\hline & & 2 & 20 & 20.1 & 43.0 & 16.3 & 18 \\
\hline \multirow{2}{*}{$\begin{array}{l}\text { Lianhua road to } \\
\text { jingjiang park }\end{array}$} & \multirow{2}{*}{979.1} & 1 & 20 & 26.9 & 19.8 & 19.7 & 21.52 \\
\hline & & 2 & 20 & 20.1 & 34.9 & 16.3 & 18 \\
\hline \multirow{2}{*}{$\begin{array}{l}\text { Jingjiang park to } \\
\text { south shanghai } \\
\text { railway station }\end{array}$} & \multirow[b]{2}{*}{1377.3} & 1 & 20 & 25.5 & 41.7 & 19 & 20.88 \\
\hline & & 2 & 20 & 20.1 & 56.9 & 16.3 & 18 \\
\hline \multirow{2}{*}{$\begin{array}{l}\text { South shanghai } \\
\text { railway station to } \\
\text { caobao road }\end{array}$} & \multirow[b]{2}{*}{1526.8} & 1 & 20 & 20.1 & 65.2 & 16.3 & 18 \\
\hline & & 2 & 20 & 20.1 & 65.2 & 16.3 & 18.04 \\
\hline \multirow{2}{*}{$\begin{array}{l}\text { Caobao road to } \\
\text { shanghai indoor } \\
\text { stadium }\end{array}$} & \multirow{2}{*}{961.2} & 1 & 20 & 20.1 & 34.1 & 16 & 18 \\
\hline & & 2 & 20 & 24.6 & 23.6 & 18.3 & 20.4 \\
\hline
\end{tabular}

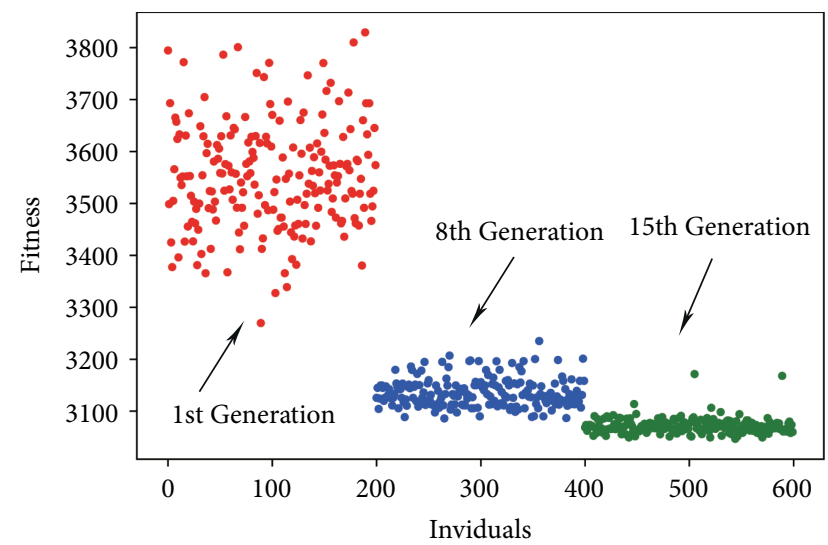

FIgURE 5: Population distribution for 1, 10, and 15 generations.

(SML1), which is one of the oldest metro lines in China. There are totally 28 stations with a daily ridership of over 1,000,000 passengers [37]. According to the statistical data in peak hours of workday, metro network implement the tight schedule, where the average travel time during a section is only $2 \mathrm{~min}$, and the uniform interval time between trains is $164 \mathrm{~s}$. At first, a simple case that two trains run over 6 sections of SML1 which from Xinzhuang to Shanghai Indoor Stadium is analyzed. Then a more complex case that three trains run over 13 sections of SML1, which is from Xinzhuang to Xinzha Road, is analysed.

Example 5.1. The first numerical example validates GA in the energy optimization problem. The experiment is set in the 6 sections of SML1. In this case, the headway is set as $120 \mathrm{~s}$ and the dwell time is set as $20 \mathrm{~s}$. Assuming two trains driving without disturbance, the energy optimization model is based on Equation (13). After 15 generations by GA, the optimal timetable is obtained in Table 1.

Figure 5 shows the population distribution of the 1st, 8 th and 15 th generations given by GA. The population distribution of the 1st generation is discrete and stochastic. After 8 iterations, the population distribution gradually centralizes. Finally after 15 generations, the population converges at a fitness value of $286.48 \mathrm{kWh}$, which is the optimal energy consumption without disturbances happen.

Example 5.2. This example is based on a two-train network in the 6 sections of SML1. A disturbance is random selected from the discrete point set of $(1 s, 2 s, \ldots, 6 s)$, and the disturbance occurs when Train No. 2 (T2) running at the 2nd station (S2) or at the 3rd station (S3). In each travel, only a disturbance happens. The optimization model is based on Equation (16). In Figure 6, indexes such as energy saving and time consumption are compared between GA and RTTRM. The initial population size of GA is set as 200. After 5, 8 and 11 generations, the population size still keeps 200 and the fitness values gradually improve. Figure 6 (left) shows the population distribution in these generations. GA_5gen, GA_8gen and GA_11gen represent the 5th, the 8th and the 11th generations offspring, respectively. Figure 6 (right) shows the calculation time in different methods.

As shown in Figure 6(a), disturbance occurs in T2S2. The 5th generation offspring of GA which distributes below zero, proposes a worse strategy than no action. By comparison, the 11th generation has a better performance, which distributed upside to the 5th generation and the 8th generation. By comparison between the results of RTTRM and the best individual of the 11th generation by GA, RTTRM averagely saves $0.840 \%$ in different delays, which is lower than GA that saves $1.115 \%$ on average. As shown in Figure 6(b), RTTRM in the T2S3 case has the best performance that saves $0.858 \%$ energy on average, which is equal to the best individual of the 11th generation by GA. Comparing from the calculation time both in the Figures 6(a) and 6(b), RTTRM has absolute advantage in order of magnitude.

Example 5.3. In this numerical example, RTTRM is validated in the three-train network in the 13 sections of SML1. In each travel test, a disturbance randomly selected from $(1 s, 2 s, \ldots, 6 s)$ occurs when the train No.1 stops at the 2 nd 

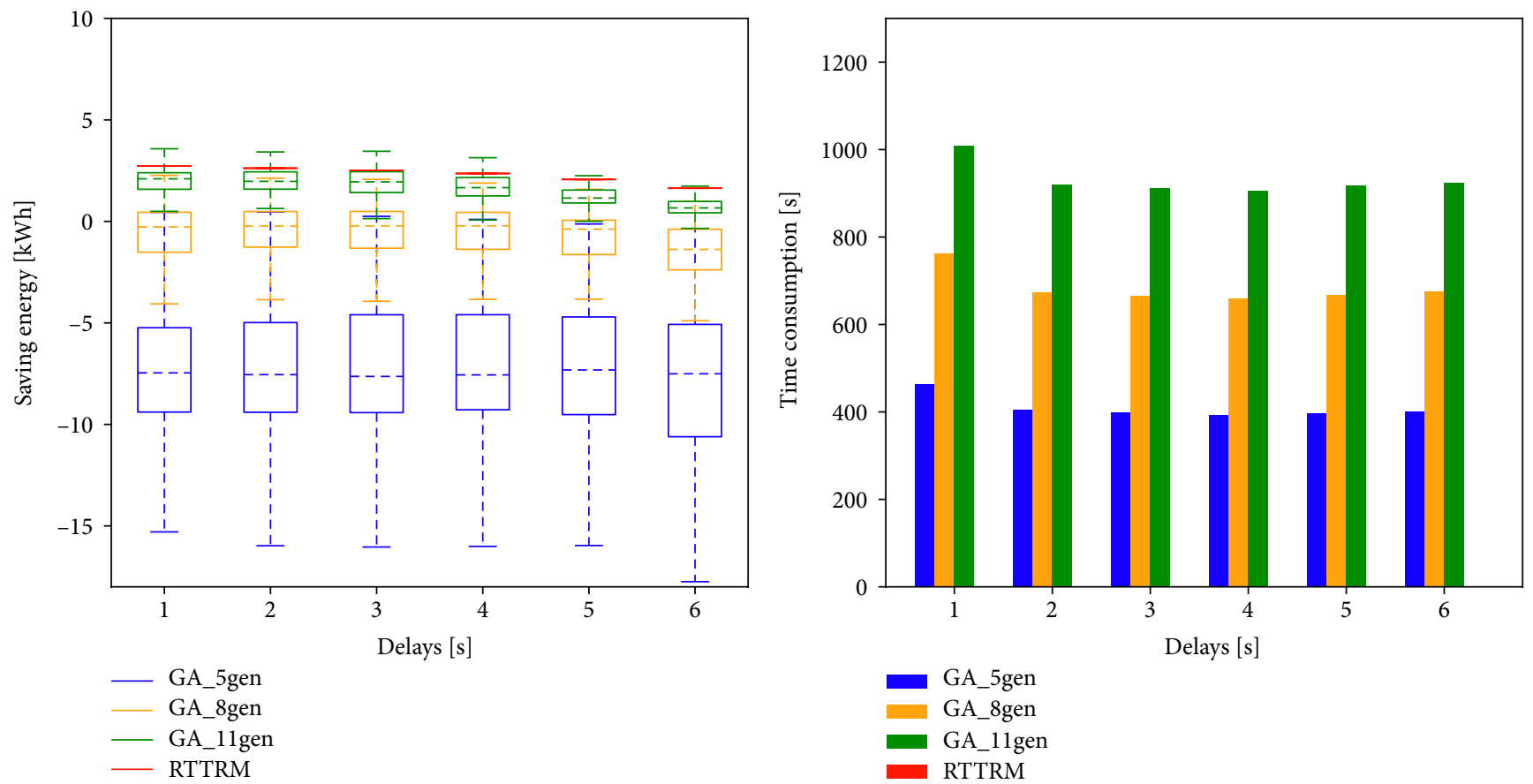

(a)
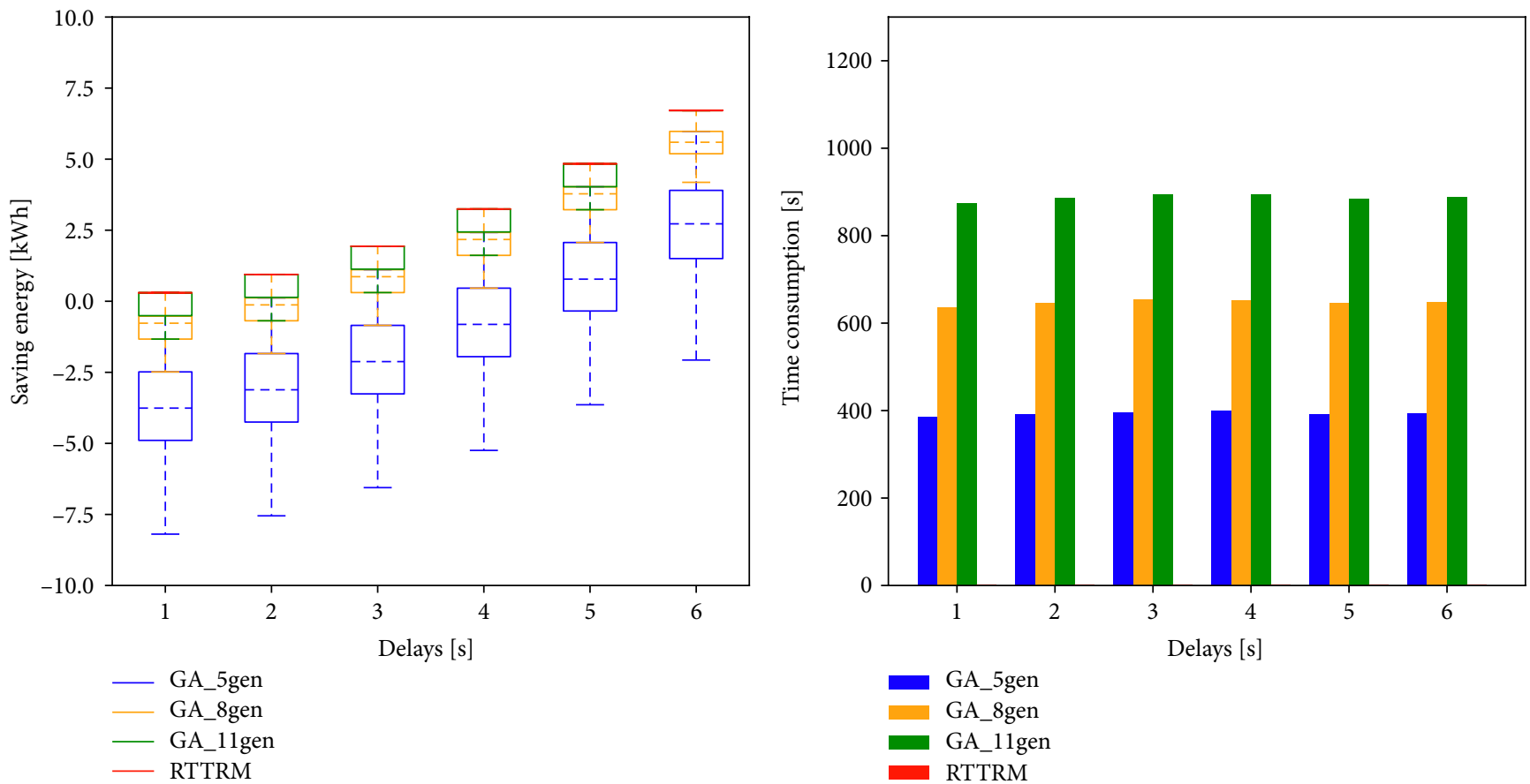

(b)

FIGURE 6: Energy saving and time consumption in two-train network. (a) Random delays occur when Train No.2 running at the 2nd station and (b) Random delays occur when Train No. 2 running at the 3rd station.

station. Both the GA and the RTTRM are used to generate the timetable after the delay happens, and then the final energy consumption of the metro system is calculated in each strategy and in each case of delay. The results are shown in Figure 7.

As can be seen from Figure 7, RTTRM can save 4.354\% energy on average in the three-train network, while GA in the 11th generation saves $3.212 \%$ energy on average, both of which save more energy percentage than the two-train network. By comparing Figures 6 and 7, it shows that to increase the number of trains in the network, more energy will be saved. According to the time consumption statistics, the calculation time to iterate a suboptimal strategy for the RTTRM is only $0.25 \mathrm{~s}$ on average. By comparison, GA takes 4500 s to reach similar effects.

Example 5.4. This numerical example is based on a threetrain network. The changing process of energy consumption during the 13 sections of SML1 among three different 

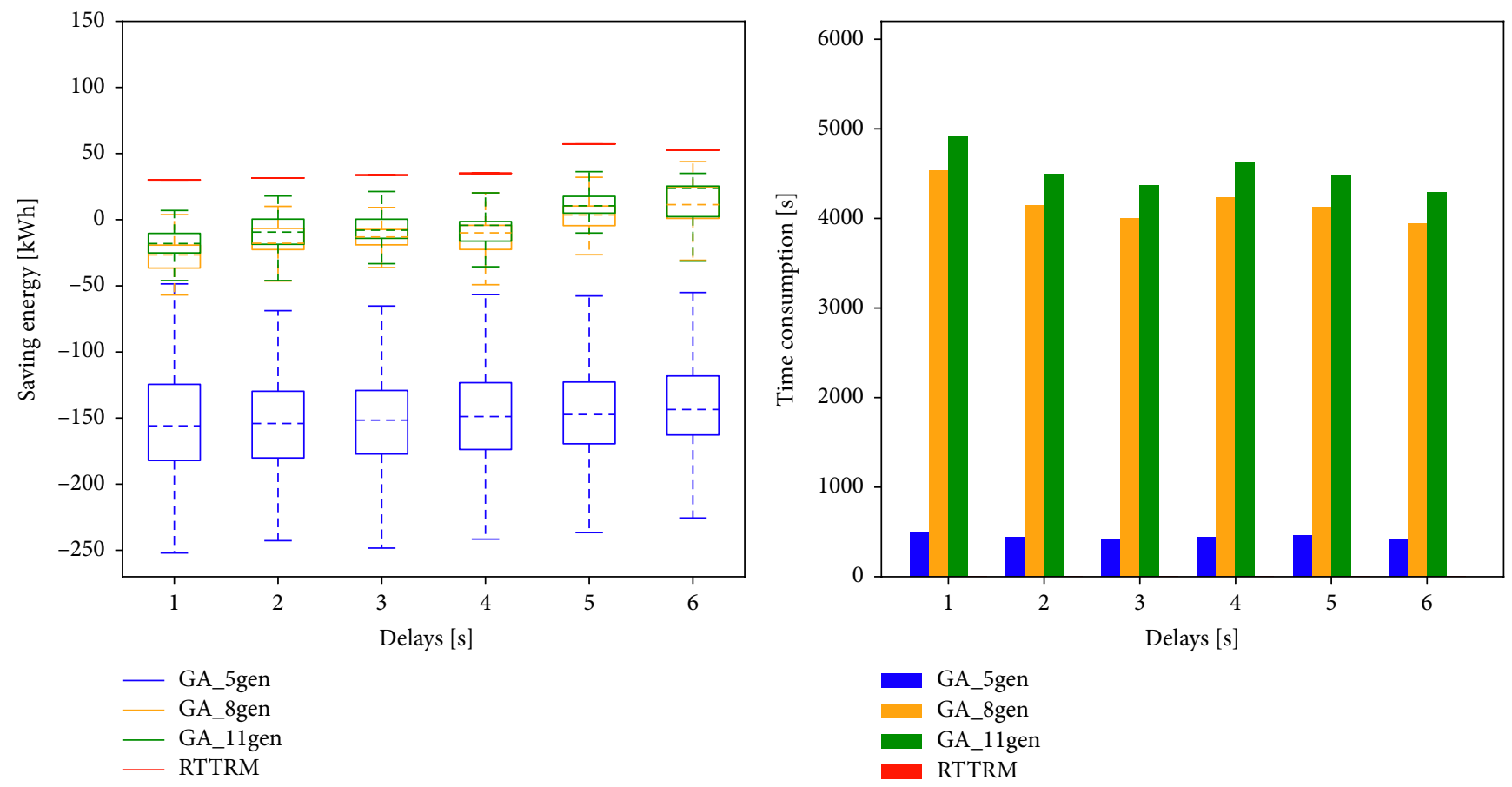

FIGURE 7: Energy saving and time consumption in three-train network.

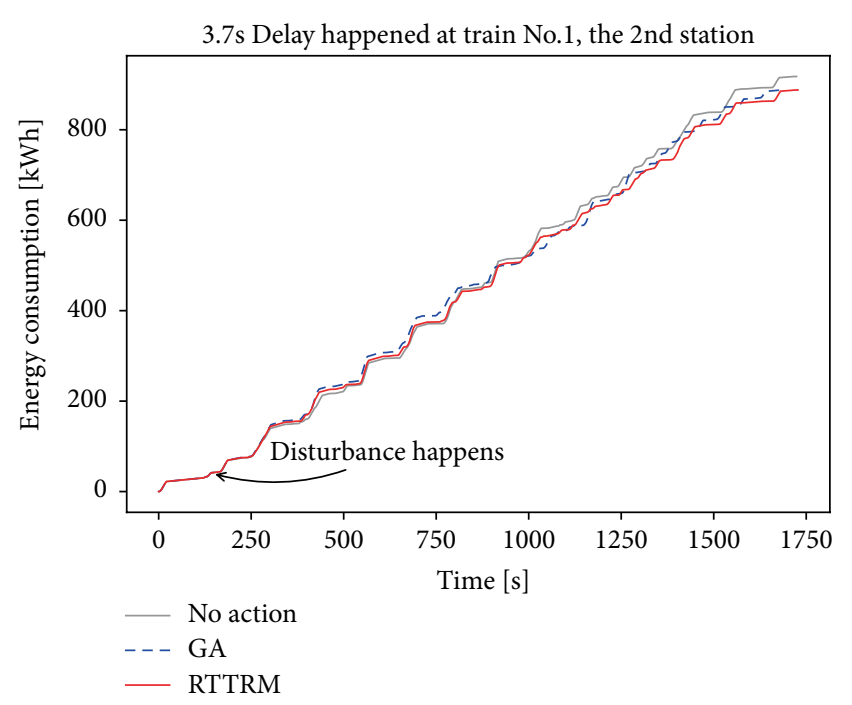

FIGURE 8: Energy consumption during the train driving process in different strategies in the case of a random delay happens.

methods is compared. The disturbance is set as $3.7 \mathrm{~s}$ when Train No.1 stops at the 2nd station. In the first method, the train does not take any action when delay occurs. In the second method, GA is used to give an offline strategy to cope with delay. In the third method, RTTRM is used to generate an optimal timetable in real time. Figure 8 gives the energy consumption of the whole journey in different methods.

As can be seen from Figure 8, the energy consumption with no-action method maintains as the highest from the moment when the delay occurs to the end. The GA method is
TABLE 2: Comparison of computation time and energy consumption among different strategies.

\begin{tabular}{lccc}
\hline Index & No action & GA & RTTRM \\
\hline $\begin{array}{l}\text { Computation time [s] } \\
\begin{array}{l}\text { Energy consumption in } \\
\text { whole journey [kWh] }\end{array}\end{array}$ & 0 & 4512.882 & 0.203 \\
\hline
\end{tabular}

proven to achieve good results in energy saving, but it takes too much time to give a decision. Compared with the two methods above, RTTRM saves $3.261 \%$ of the total energy in this case, and can fulfill the real-time requirement in the energy-efficient TTR problem.

Table 2 gives the computation time and the total energy consumption of these methods. The computation time is measured in the CPU Intel i7-4720HQ.

\section{Conclusion}

In this paper, a real-time timetable rescheduling method for minimizing the overall energy consumption of metro systems under disturbances is proposed. Different from the prevailing methods for TTR problem focusing on minimizing the overall delay, the method focuses on solving energy-efficient TTR problems that maximizing the usage of regenerative energy. The difficulties of energy-efficient TTR problems are the demand of real-time response and the randomness of disturbances. Combining GA and DNN, the proposed method can reschedule the timetable in a very short response time and have a self-adaptability that is able to make decisions for different cases of disturbances. The method is based on three 
models: a control model, a timetable model, and an energy model. The energy model formulates the optimization equations in both cases with and without disturbances. The randomness of dwelling-time disturbances is described in the energy model. The most important part of the method is a GA optimizer and a decision system. GA is used to search an optimal timetable for each case of the delay in order to minimize the energy consumption as well as extracting the state variables in each optimal strategy into a training set. The decision system based on DNN is proposed to give a wise advice for the cruising speed according to the state variables samples from GA.

Several experimental studies are conducted on Shanghai Metro Line 1 to validate the method. Both two-train network and three-train network are simulated in the experiments. The results indicate that the decision system is effective to select suboptimal cruising speed for the energy saving concern. RTTRM saves $0.840 \%$ energy on average in two-train network in the 6 sections of SML1, and saves $4.354 \%$ energy on average in three-train network in the 13 sections of SML1. The method only takes $0.010 \mathrm{~s}$ in the two-train network and $0.25 \mathrm{~s}$ in threetrain network on average, which is much faster than GA. A whole journey comparison between RTTRM and GA is done under a $3.7 \mathrm{~s}$ disturbance situation. RTTRM saves $3.261 \%$ energy, which is almost equal to GA, but the computation time of the RTTRM is only $0.203 \mathrm{~s}$.

\section{Appendix}

\section{Derivation Process}

According to Equations (1) and (2), the equation is deduced as:

$$
\begin{gathered}
\frac{d t}{d v}=\frac{1}{u-r+g}, \\
\frac{d x}{d v}=\frac{d x}{d t} \cdot \frac{d t}{d v}=\frac{v}{u-r+g} .
\end{gathered}
$$

Integral Equation (A.2) as

$$
x=\int \frac{v}{u-r+g} d v .
$$

The distance in each phase is calculated as:

$$
\begin{gathered}
x_{1}^{\eta, \gamma}=\int_{0}^{v_{a}} \frac{v}{u_{a}-r+g} d v, \\
x_{2}^{\eta, \gamma}=\int_{v_{a}}^{v_{c}^{\eta, \gamma}} \frac{v}{\left(u_{a} v_{a} / v\right)-r+g} d v, \\
x_{4}^{\eta, \gamma}=\int_{v_{c}^{\eta, \gamma}}^{v_{b}} \frac{v}{\left(u_{b} v_{b} / v\right)-r+g} d v, \\
x_{5}^{\eta, \gamma}=\int_{v_{b}}^{0} \frac{v}{u_{b}-r+g} d v, \\
x_{3}^{\eta, \gamma}=x_{s}^{\eta}-x_{1}^{\eta, \gamma}-x_{2}^{\eta, \gamma}-x_{4}^{\eta, \gamma}-x_{5}^{\eta, \gamma},
\end{gathered}
$$

where $\left(x_{1}^{\eta, \gamma}, x_{2}^{\eta, \gamma}, x_{3}^{\eta, \gamma}, x_{4}^{\eta, \gamma}, x_{5}^{\eta, \gamma}\right)$ represent the running distance in the five phases, which are the constant force accelerating phase, the constant power accelerating phase, the speedhold phase, the constant power braking phase and the constant force braking phase. So the function $t_{s}^{\eta, \gamma}=f\left(v_{c}^{\eta, \gamma}\right)$ can be expressed as:

$$
\begin{aligned}
t_{s}^{\eta, \gamma}= & \int_{0}^{v_{a}} \frac{1}{u_{a}-r+g} d v+\int_{v_{a}}^{v_{c}^{\eta, \gamma}} \frac{1}{\left(u_{a} v_{a} / v\right)-r+g} d v \\
& +\int_{v_{c}^{\eta, \gamma}}^{v_{b}} \frac{1}{\left(u_{b} v_{b} / v\right)-r+g} d v+\int_{v_{b}}^{0} \frac{1}{u_{b}-r+g} d v+\frac{x_{3}^{\eta, \gamma}}{v_{c}^{\eta, \gamma}} .
\end{aligned}
$$

\section{Data Availability}

The data used to support the findings of this study are included within the article.

\section{Conflicts of Interest}

The authors declare that they have no conflicts of interest.

\section{Acknowledgments}

The authors would like to acknowledge the fund supported by the Shanghai Shentong Metro Group Co., Ltd., which also provides experimental environment and assistance. The article was funded by Shanghai Shentong Metro Group Co., Ltd, grant number No. JS-372 KY09R013.

\section{References}

[1] H. Dong, B. Ning, B. Cai, and Z Hou, "Automatic train control system development and simulation for high-speed railways," IEEE Circuits and Systems Magazine, vol. 10, no. 2, pp. 6-18, 2010.

[2] X. Li, B. Shou, and R. Dan, "Train rescheduling with stochastic recovery time: a new track-backup approach," IEEE Transactions on Systems Man \& Cybernetics Systems, vol. 44, no. 9, pp. 12161233, 2017.

[3] S. Binder, Y. Maknoon, and M. Bierlaire, "The multi-objective railway timetable rescheduling problem," Transportation Research Part C Emerging Technologies, vol. 78, pp. 78-94, 2017.

[4] F. A. Ortega, M. A. Pozo, and J. Puerto, "On-line timetable rescheduling in a transit line," Transportation Science, vol. 52, no. 5, pp. 1106-1121, 2018.

[5] L. Wang, W. Mo, Y. Qin, F. Dou, and L. Jia, "Optimization based high-speed railway train rescheduling with speed restriction," Discrete Dynamics in Nature and Society, vol. 2014, Article ID 934369, p. 14, 2014.

[6] W. Xu, P. Zhao, and L. Ning, "A practical method for timetable rescheduling in subway networks during the end-of-service period," Journal of Advanced Transportation, vol. 2018, Article ID 5914276, pp. 1-9, 2018.

[7] P. Xu, F. Corman, Q. Peng, and X. Luan, "A timetable rescheduling approach and transition phases for high-speed railway traffic during disruptions," Transportation Research Record Journal of the Transportation Research Board, vol. 2607, no. 1, pp. 82-92, 2017. 
[8] P. Dalapati, P. Agarwal, A. Dutta, and S. Bhattacharya, "Realtime rescheduling in distributed railway network: an agentbased approach," 2016, https://arxiv.org/abs/1607.03340.

[9] G. M. Scheepmaker, R. M. P. Goverde, and L. G. Kroon, "Review of energy-efficient train control and timetabling," European Journal of Operational Research, vol. 257, no. 2, pp. 355-376, 2017.

[10] C. Gong, S. Zhang, F. Zhang, J. Jiang, and X. Wang, "An integrated energy-efficient operation methodology for metro systems based on a real case of shanghai metro line one," Energies, vol. 7, no. 11, pp. 7305-7329, 2014.

[11] M. Carey, "Reliability of interconnected scheduled services," European Journal of Operational Research, vol. 79, no. 1, pp. 51-72, 1994.

[12] M. Carey and A. Kwieciński, "Stochastic approximation to the effects of headways on knock-on delays of trains," Transportation Research Part B: Methodological, vol. 28, no. 4, pp. 251-267, 1994.

[13] M. Carey and A. Kwieciński, "A properties of expected costs and performance measures in stochastic models of scheduled transport," European Journal of Operational Research, vol. 83, no. 1, pp. 182-199, 1994.

[14] J. Törnquist and J. A. Persson, "N-tracked railway traffic rescheduling during disturbances," Transportation Research Part B Methodological, vol. 41, no. 3, pp. 342-362, 2007.

[15] A. D’Ariano, D. Pacciarelli, and M. Pranzo, "A branch and bound algorithm for scheduling trains in a railway network," European Journal of Operational Research, vol. 183, no. 2, pp. 643-657, 2007.

[16] M. B. Khan and X. Zhou, "Stochastic optimization model and solution algorithm for robust double-track train-timetabling problem," IEEE Transactions on Intelligent Transportation Systems, vol. 11, no. 1, pp. 81-89, 2010.

[17] V. Cacchiani and P. Toth, "Nominal and robust train timetabling problems," European Journal of Operational Research, vol. 219, no. 3, pp. 727-737, 2012.

[18] L. Kroon, G. Maróti, M. R. Helmrich, M. Vromans, and R. Dekker, "Stochastic improvement of cyclic railway timetables," Transportation Research Part B: Methodological, vol. 42, no. 6, pp. 553-570, 2008.

[19] R. K. Ahuja, R. H. Möhring, and D. Z. Christos, Robust and Online Large-Scale Optimization: Models and Techniques for Transportation Systems, vol. 5868, Springer, 2009.

[20] S. Cicerone, G. D’Angelo, G. Di Stefano, D. Frigioni, and A. Navarra, "Recoverable robust timetabling for single delay: complexity and polynomial algorithms for special cases," Journal of Combinatorial Optimization, vol. 18, no. 3, pp. 229-257, 2009.

[21] C. Liebchen, M. Schachtebeck, A. Schöbel, S. Stiller, and A. Prigge, "Computing delay resistant railway timetables," Computers \& Operations Research, vol. 37, no. 5, pp. 857-868, 2010.

[22] V. Cacchiani, A. Caprara, and M. Fischetti, "A lagrangian heuristic for robustness with an application to train timetabling," Transportation Science, vol. 46, no. 1, pp. 124-133, 2012.

[23] M. Tormos, A. Lova, Ingolotti, and L. P., "A genetic approach to robust train timetabling, technical report ARRIVAL-TR-0173 arrival project," 2008.

[24] S. Dündar and I. Şahin, "Train re-scheduling with genetic algorithms and artificial neural networks for single-track railways," Transportation Research Part C: Emerging Technologies, vol. 27, no. 2, pp. 1-15, 2013.

[25] D. Šemrov, R. Marsetič, M. Žura, L. Todorovski, and A. Srdic, "Reinforcement learning approach for train rescheduling on a single-track railway," Transportation Research Part B: Methodological, vol. 86, pp. 250-267, 2016.

[26] T. Albrecht and S. Oettich, "A new integrated approach to dynamic schedule synchronization and energy-saving train control," WIT Transactions on The Built Environment, vol. 61, 2002.

[27] T. Albrecht, "Reducing power peaks and energy consumption in rail transit systems by simultaneous train running time control," Computers in Railways, vol. 769, no. 769, pp. 100-107, 2004.

[28] X. Yang, X. Li, Z. Gao, H. Wang, and T. Tang, "A cooperative scheduling model for timetable optimization in subway systems," IEEE Transactions on Intelligent Transportation Systems, vol. 14, no. 1, pp. 438-447, 2013.

[29] H. Sun, J. Wu, H. Ma, X. Yang, and Z. Gao, "A bi-objective timetable optimization model for urban rail transit based on the time-dependent passenger volume," IEEE Transactions on Intelligent Transportation Systems, vol. 20, no. 2, pp. 604-615, 2018.

[30] W. J. Davis, "The tractive resistance of electric locomotives and cars," General Electric, 1926.

[31] A. Albrecht, P. Howlett, P. Pudney, X. Vu, and P. Zhou, “The key principles of optimal train control part 1: Formulation of the model, strategies of optimal type, evolutionary lines, location of optimal switching points," Transportation Research Part B: Methodological, vol. 94, pp. 482-508, 2016.

[32] V. Van Breusegem, G. Campion, and G. Bastin, "Traffic modeling and state feedback control for metro lines," IEEE Transactions on Automatic Control, vol. 36, no. 7, pp. 770-784, 1991.

[33] J. H. Holland, "Adaptation in natural and artificial systems: An introductory analysis with applications to biology, control, and artificial intelligence," Ann Arbor, vol. 6, no. 2, pp. 126-137, 1975.

[34] GitHub Inc, "DEAP," 2019, https://github.com/deap/deap.

[35] Wikepedia, “Deap learning," 2019, https://en.wikipedia.org/ wiki/Deep_learning.

[36] M. Bianchini and F. Scarselli, "On the complexity of neural network classifiers: a comparison between shallow and deep architectures," IEEE Transactions on Neural Networks \& Learning Systems, vol. 25, no. 8, pp. 1553-1565, 2014.

[37] Wikipedia, "Line1(Shanghai Metro)", 2018, https://en.wikipedia. org/wiki/Line_1_(Shanghai_Metro). 


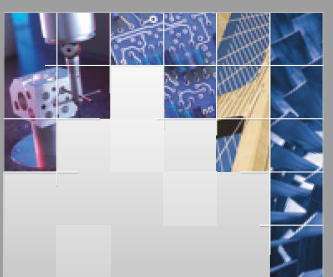

\section{Enfincering}
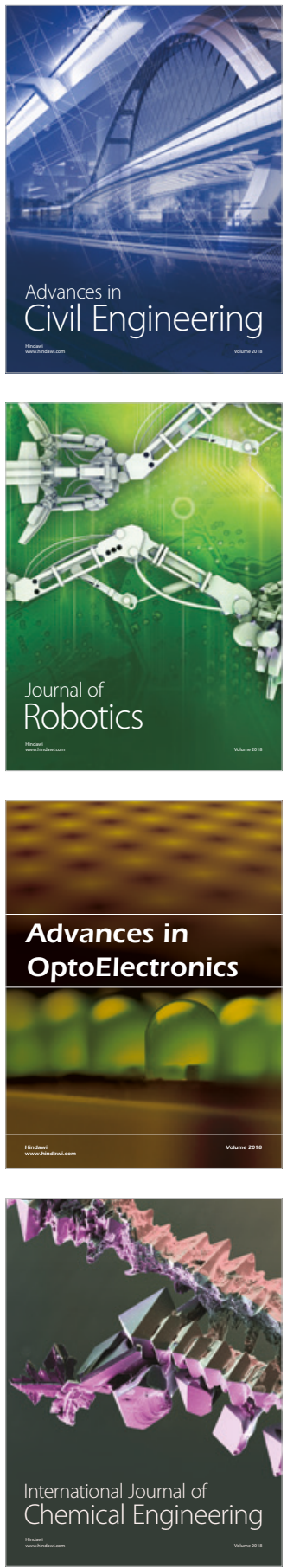

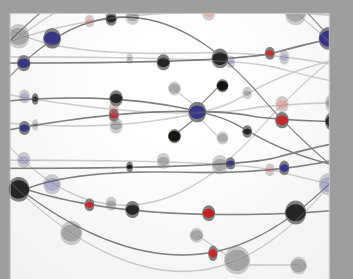

\section{Rotating \\ Machinery}

The Scientific World Journal

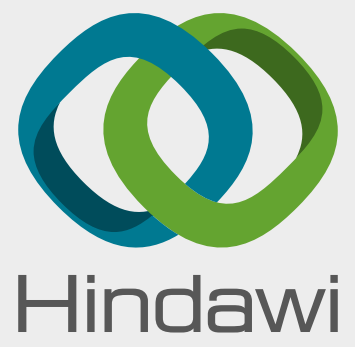

Submit your manuscripts at

www.hindawi.com
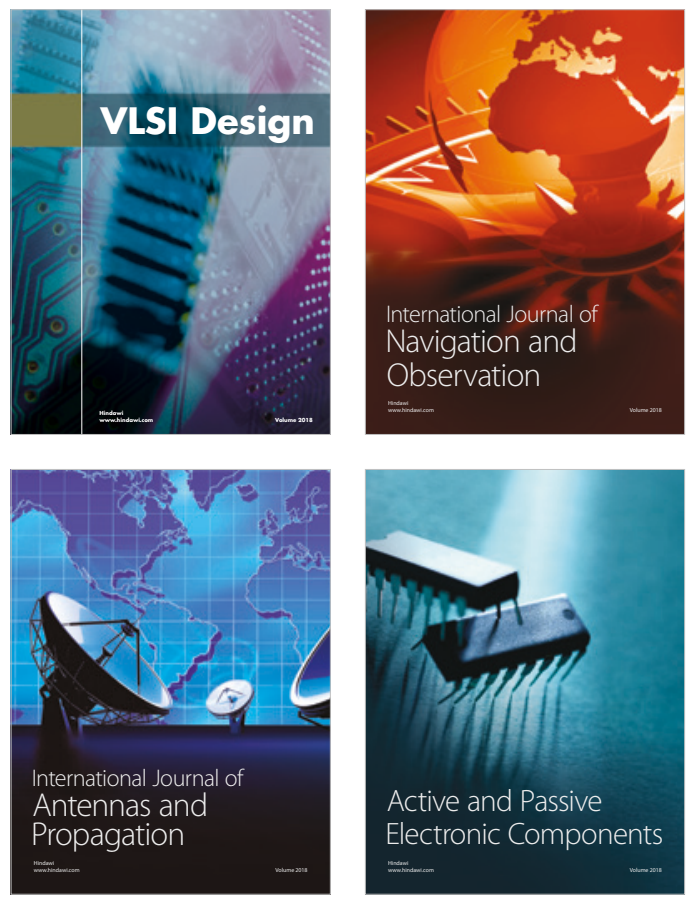
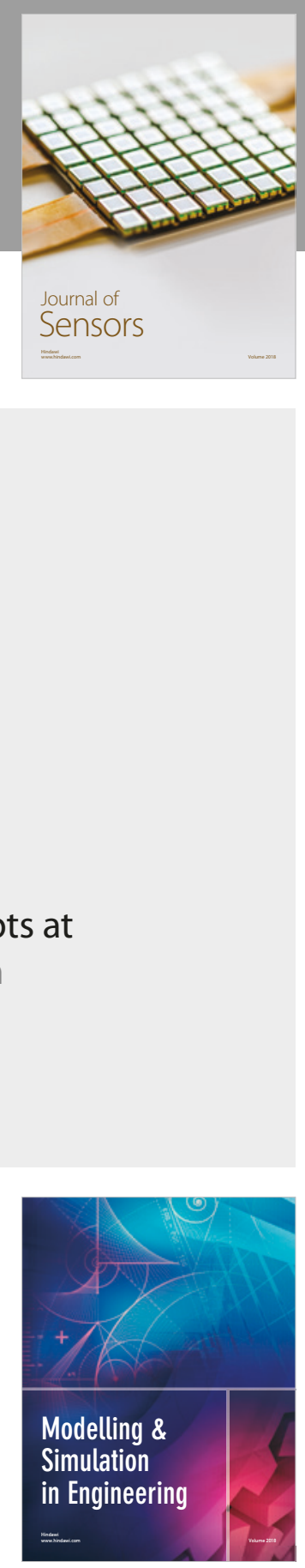

\section{Advances \\ Multimedia}
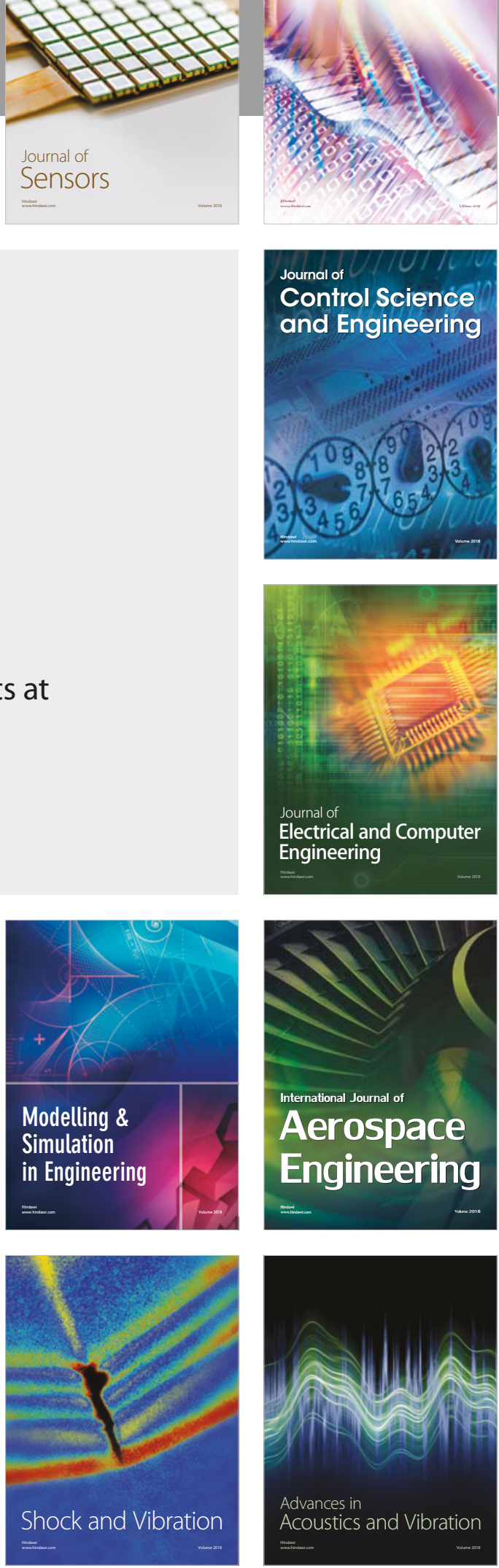